\title{
Analytical Model for High Impedance Fault Analysis in Transmission Lines
}

\author{
S. Maximov, V. Torres, H. F. Ruiz, and J. L. Guardado \\ Instituto Tecnológico de Morelia, Avenida Tecnológico No. 1500, 58120 Morelia, MICH, Mexico \\ Correspondence should be addressed to V. Torres; vtg_1982@hotmail.com
}

Received 6 May 2014; Accepted 27 August 2014; Published 11 September 2014

Academic Editor: Ebrahim Momoniat

Copyright (C) 2014 S. Maximov et al. This is an open access article distributed under the Creative Commons Attribution License, which permits unrestricted use, distribution, and reproduction in any medium, provided the original work is properly cited.

\begin{abstract}
A high impedance fault (HIF) normally occurs when an overhead power line physically breaks and falls to the ground. Such faults are difficult to detect because they often draw small currents which cannot be detected by conventional overcurrent protection. Furthermore, an electric arc accompanies HIFs, resulting in fire hazard, damage to electrical devices, and risk with human life. This paper presents an analytical model to analyze the interaction between the electric arc associated to HIFs and a transmission line. A joint analytical solution to the wave equation for a transmission line and a nonlinear equation for the arc model is presented. The analytical model is validated by means of comparisons between measured and calculated results. Several cases of study are presented which support the foundation and accuracy of the proposed model.
\end{abstract}

\section{Introduction}

High impedance faults are those that do not produce enough current to be detected in a reliable way by conventional devices such as relays [1]. HIF detection and localization in electrical power systems has been traditionally a challenge for protection engineers. This is due to the nature of this kind of faults, basically their variability and relatively low-current levels compared to substation load currents. Furthermore, arcing accompanies HIF, resulting in fire hazard, damage to electrical devices, and risk to human life. Under these circumstances, conventional protection relays are unable to detect and locate such faults. Though many HIFs do not involve ground at all (phase-to-phase faults due to leaning trees), phase-to-ground faults are of paramount interest because of their relevance for public safety.

This has long been recognized by the industry and since the early $70 \mathrm{~s}$, several methods have been proposed in the literature for HIF detection. They are based on examining different characteristics of currents and voltages in the time, frequency, and time-frequency domains. Some of these techniques are the staged fault test [2], low frequency spectrum [3], Kalman filtering [4], neural networks [5], neural networks and wavelet [6], expert systems [7], and more recently the application of harmonics analysis $[8,9]$, the wavelet transform [10-12], and the correlation function [13].

For HIF fault localization, techniques such as numerical algorithms [14], nonlinear frequency analysis [15], and recently, time domain studies [16] have been proposed. However, the authors consider that a complete solution to the problem of HIF detection and localization can be achieved only by a deep understanding of the interaction between the HIF and the transmission line.

In this paper, a new model to analyze the interaction between a lossless transmission line and the electric arc associated during a HIF is proposed. The transmission line is represented by the nondissipative and nondispersive wave equation and the electric arc is modelled by a Mayr-Cassie type equation. The novelty and the mathematical challenge of this problem consist in the joint solution of a linear partial differential equation, which is the wave equation, and an essentially nonlinear ordinary differential equation for the arc model. The nonlinearity of the arc phenomenon yields a general impossibility of considering the electric arc effect as load impedance in the analytic form. An elegant solution to this problem is proposed by showing that for a wide class of periodic voltages the arc phenomenon can be represented like impedance which depends on voltage 
frequency. As a result, the problem becomes formally linear and can be solved analytically. Thus, the nonlinearity of the phenomenon is "hidden" in the impedance dependence on the wave frequency.

The authors expect to make a contribution to the understanding of the complex phenomenon of HIFs interacting with a power transmission line. The analysis and results presented along the paper also laid the ground for the development of future techniques for HIF detection and localization on power and distribution lines.

\section{High Impedance Fault Model}

2.1. Electric Arc Model. The development of a methodology for HIF localization and detection requires a suitable representation for the high impedance existing during this kind of phenomenon. In this paper, a model based in the electric arc is proposed for representing this impedance.

Despite the complexity associated with the arc behavior, different methods have been developed to model and simulate its behavior. Thermal models are commonly used to describe the electric arc dynamics. These models have a long history since Cassie and Mayr $[17,18]$ introduced their first descriptions of the arc conductivity in the form of a firstorder differential equation. These dynamic equations have been improved and modified in order to increase the models validity and to reduce the computational burden. Most of such equations are of the form $[19,20]$

$$
\frac{d \ln g}{d t}=\frac{1}{\tau(v, i)}\left(\frac{v i}{P(v, i)}-1\right),
$$

where $g=i / v, v$, and $i$ are the arc conductance, arc voltage, and arc current, respectively, $\tau(v, i)$ is the time constant, and $P(v, i)$ is the cooling power. In the high- and lowcurrent regions, (1) turns into the Mayr and Cassie models, respectively [19]. In [21] Kizilcay and Pniok proposed a model which can be obtained from the general equation (1) by taking $\tau$ as a constant and the heat dissipation power in the form $P=P_{0}+V_{0}|i|$. As a result the arc conductance equation takes the form

$$
\frac{d g}{d t}=\frac{G(i)-g}{\tau}
$$

where the steady state conductance is a function of the arc current:

$$
G(i)=\frac{i^{2}}{P_{0}+V_{0}|i|} .
$$

First, if the electric arc current in (3) is of the sinusoidal (or quasi-sinusoidal) form with frequency $\omega=2 \pi / T$, then, according to (3), the steady state conductance is a periodic function with frequency $2 \omega$, which can be expanded in Fourier series with the even harmonics:

$$
G(t)=\sum_{n=-\infty}^{\infty} G_{n} e^{j 2 n \omega t} .
$$

If the period of (3) $\tau \gg(T / 2)$, then the steady state conductance is the superposition of the constant part $G_{0}$ and the rapidly oscillating part $\delta G(t)$ :

$$
G(t)=G_{0}+\delta G(t),
$$

where the rapidly oscillating part can be filtered by the operation:

$$
\bar{G}(t)=\frac{1}{T} \int_{0}^{T} G(t) d t=G_{0} .
$$

Then, substituting (5) into (2), the arc conductance $g(t)$ can be represented as a sum of the "slow" part $\bar{g}(t)$, which does not change significantly during one period $T$ and the rapidly oscillating component $\delta g(t)$ is as follows:

$$
g(t)=\bar{g}(t)+\delta g(t) .
$$

The equation for the slow part of the arc conductance can be obtained by applying the averaging operation (6) to both parts of (2). Then, due to the linearity of the operator (6),

$$
\frac{d \bar{g}}{d t}=\frac{G(i)-\bar{g}}{\tau}
$$

and the solution to this equation in the time domain has the form

$$
\bar{g}(t)=G_{0}\left(1-e^{-t / \tau}\right) .
$$

This paper only considers the slow part of the arc conductance and how this part interacts with the transmission line. A more exact description requires considering the full Fourier series (4). Evidently, the rapidly oscillating part of the steady state conductance injects harmonics into the transmission line. The analysis of such harmonics is an interesting problem, which can provide the complete information about the HIF. However, this problem is beyond the purpose of this paper.

In the next subsection, (9) is validated by obtaining the arc model parameters $G_{0}$ and $\tau$ from measurements.

2.2. Parameter Calculation for the Arc Model. In this section, field measurements during HIFs are used to calculate the arc model parameters $G_{0}$ and $\tau$. The HIF records were obtained during field test in a $13.8 \mathrm{kV}$ distribution circuit owned by the national utility of México (CFE). Figures 1 and 2 show the current and voltage waveforms, respectively, during a HIF.

The parameters $G_{0}$ and $\tau$ can be obtained from the current and voltage waveforms by using the least square method. Let us denote the fault conductance, current, and voltage by $\bar{g}_{f}\left(t_{k}\right), i_{f}\left(t_{k}\right)$, and $v_{f}\left(t_{k}\right)$, respectively, taken at discrete time instants $t_{1}, t_{2}, \ldots, t_{N}$.

The averaged ("slow") arc conductance can be calculated as follows:

$$
\bar{g}_{f}\left(t_{k}\right)=\sqrt{\frac{\sum_{i=-n}^{n} i_{f}^{2}\left(t_{k+i}\right)}{\sum_{i=-n}^{n} v_{f}^{2}\left(t_{k+i}\right)}},
$$

where the number $n$ is taken large enough to smooth the conductance curve. $\bar{g}\left(t_{k}\right)$ is the fault conductance predicted 


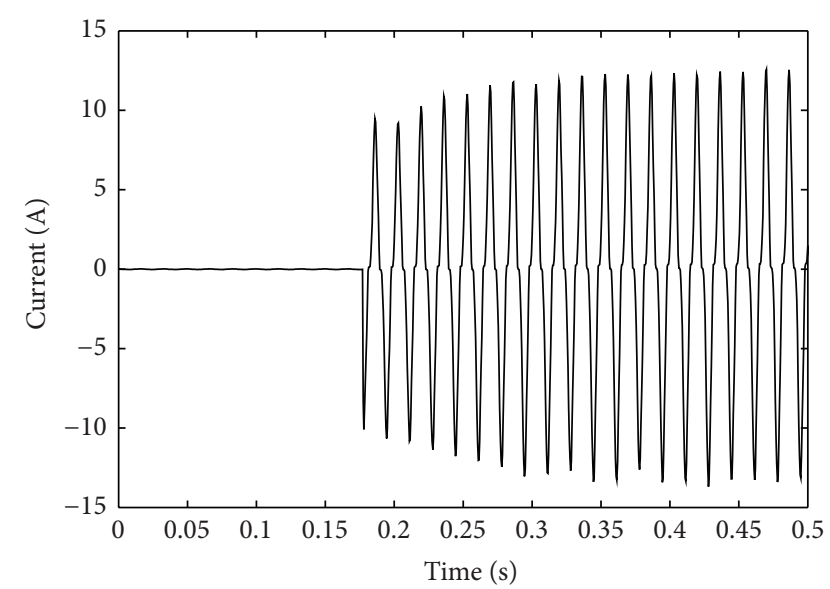

FIGURE 1: Measured current during a high impedance fault.

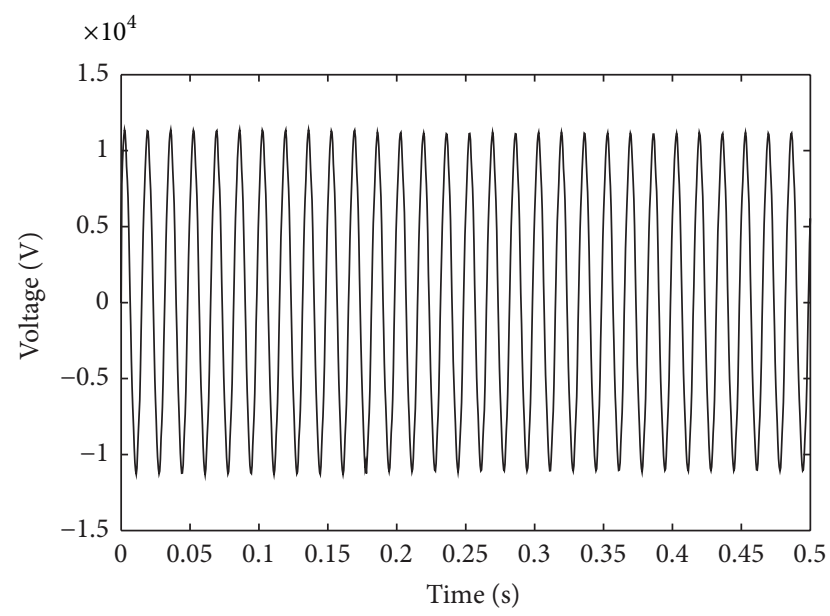

FIGURE 2: Measured voltage during a high impedance fault.

by the model (9) at discrete moments $t_{k}$. Then, the sum of squared deviations of the theoretical values from the experimental data is

$$
S\left(G_{0}, \tau\right)=\sum_{k=1}^{N}\left(\bar{g}\left(t_{k}\right)-\bar{g}_{f}\left(t_{k}\right)\right)^{2} .
$$

The minimum of the sum of squares is found by solving the following system of equations:

$$
\frac{\partial\left(S\left(G_{0}, \tau\right)\right)}{\partial G_{0}}=0, \quad \frac{\partial\left(S\left(G_{0}, \tau\right)\right)}{\partial \tau}=0 .
$$

This system of equations can be solved numerically by any suitable method, for example, by the gradient descent technique [19]. The results of solving the system (12) are $G_{0}=0.000065 \mho$ and $\tau=0.051 \mathrm{~s}$. The validation of these parameter calculations will be shown in Section 6 .

\section{Lossless Transmission Line}

Figure 3 shows a single-phase two-wire lossless transmission line of length $l$, a source $V_{S}$ with impedance $Z_{S}$ at the sending

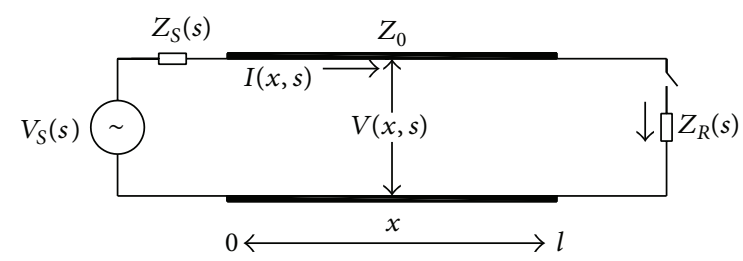

FIGURE 3: Transmission line terminated on a HIF.

end and terminated by an impedance $Z_{R}$, associated with the HIF.

The equations that describe the lossless transmission line with distributed parameter are

$$
\begin{aligned}
& \frac{\partial v(x, t)}{\partial x}=-L \frac{\partial i(x, t)}{\partial t}, \\
& \frac{\partial i(x, t)}{\partial x}=-C \frac{\partial v(x, t)}{\partial t}
\end{aligned}
$$

where $L$ and $C$ are the transmission line inductance and capacitance, respectively. Equations (13) and (14) can be reduced to the so-called travelling wave equation for voltage [20]:

$$
\frac{\partial^{2} v(x, t)}{\partial x^{2}}-\frac{1}{c^{2}} \frac{\partial^{2} v(x, t)}{\partial t^{2}}(x, t)=0
$$

where $c$ is the wave velocity:

$$
c=\frac{1}{\sqrt{L C}} .
$$

The general solution to (15) in the Laplace domain has the form

$$
\begin{aligned}
V(x, s)= & A(s) e^{-s x / c}+B(s) e^{s x / c} \\
& -\frac{1}{s c} \int_{0}^{x} \sinh \left(\frac{s}{c}(x-y)\right)\left(v_{t}(y, 0)+s v(y, 0)\right) d y .
\end{aligned}
$$

Equation (15) should be considered together with the respective boundary conditions:

$$
\begin{aligned}
& V(0, s)=V_{S}(s)-Z_{S}(s) I(0, s), \\
& V(l, s)=Z_{R}(s) I(l, s) .
\end{aligned}
$$

Boundary conditions (18) contain the unknown currents in the sending and receiving ends $I(0, s)$ and $I(l, s)$, which can be found by substituting the general solution (17) into (13) in the Laplace domain. Then, the unknown terms $A(s)$ and $B(s)$ 
can be excluded from the solution (17) by using the boundary conditions (18). As a result it can be obtained as

$$
\begin{aligned}
V(x, s)= & \frac{e^{(-s / c) x}+\Gamma_{R}(s) e^{(-s / c)(2 l-x)}}{1-\Gamma_{S}(s) \Gamma_{R}(s) e^{-2 s l / c}} \\
& \times\left(\frac{V_{S}(s)\left(1-\Gamma_{S}(s)\right)}{2}-\frac{\left(1+\Gamma_{S}(s)\right) Z_{0} i(0,0)}{2 s}\right) \\
& +\frac{\Gamma_{S}(s) e^{-s x / c}+e^{s x / c}}{1-\Gamma_{S}(s) \Gamma_{R}(s) e^{-2 s l / c}} \\
& \times\left(\frac{e^{(-s / c) l}\left(1+\Gamma_{R}(s)\right) Z_{0} i(l, 0)}{2 s}+\frac{1}{2 s c}\right. \\
& \times \int_{0}^{l}\left(e^{-s y / c}+\Gamma_{R}(s) e^{(-s / c)(2 l-y)}\right) \\
& -\frac{1}{s c} \int_{0}^{x} \sin h\left(\frac{s(x-y)}{c}\right)\left(v_{t}(y, 0)+s v(y, 0)\right) d y,
\end{aligned}
$$

where $Z_{0}$ is the characteristic impedance of the line:

$$
Z_{0}=\sqrt{\frac{L}{C}}
$$

$\Gamma_{S}$ and $\Gamma_{R}$ are the reflection coefficients at the source and the receiving end, respectively:

$$
\begin{gathered}
\Gamma_{S}(s)=\frac{Z_{S}(s)-Z_{0}}{Z_{S}(s)+Z_{0}}, \\
\Gamma_{R}(s)=\frac{Z_{R}(s)-Z_{0}}{Z_{R}(s)+Z_{0}} .
\end{gathered}
$$

Solution (19) describes the traveling wave voltage in a lossless transmission line of a length $l$ with the sending and backward reflection coefficients $\Gamma_{S}$ and $\Gamma_{R}$, respectively.

For a realistic HIF modeling, the transmission line equations (13)-(15) should be considered together with the arc model equation (1) or (2). As a result, a system of a partial differential equation (15) and an ordinary nonlinear differential equation of the type (2) is obtained. Solving such a system of equations in general form is a real mathematical challenge due to the nonlinearity of (2). However, the assumptions and developments made in the previous section regarding (2) have led to a more suitable representation to the arc conductance like in (9).

\section{Modeling the Electric Arc Reflection Coefficient}

The first step for modeling the interaction between the transmission line and the HIF is to obtain the electric arc impedance $Z_{R}(s)$ and the corresponding reflection coefficient
$\Gamma_{R}(s)$. This is carried out taking into account the fact that the arc conductance $\bar{g}(t)$ changes slowly with respect to the arc voltage and current. Applying the Laplace transform to Ohm's law,

$$
i_{R}(t)=\bar{g}(t) v_{R}(t)
$$

and by using the periodicity of the arc voltage

$$
\begin{aligned}
i_{R}(s) & =\int_{0}^{\infty} e^{-s t} \bar{g}(t) v_{R}(t) d t \\
& =\sum_{n=0}^{\infty} e^{-s T n} \int_{0}^{T} e^{-s \xi} \bar{g}(T n+\xi) v_{R}(\xi) d \xi \\
& =\sum_{n=0}^{\infty} e^{-s T n} \bar{g}\left(T n+\xi_{n}^{*}\right) \Psi(s, T),
\end{aligned}
$$

where according to the mean value theorem $\xi_{n}^{*} \in[0, T]$, then

$$
\Psi(s, T)=\int_{0}^{T} e^{-s \xi} v_{R}(\xi) d \xi .
$$

On the other hand, the arc voltage in the Laplace domain is

$$
\begin{aligned}
& V_{R}(s)= \int_{0}^{\infty} e^{-s t} v_{R}(t) d t=\sum_{n=0}^{\infty} e^{-s T n} \int_{0}^{T} e^{-s \xi} v_{R}(\xi) d \xi \\
&= \frac{\Psi(s, T)}{\left(1-e^{-s T}\right)}, \\
& \Psi(s, T)=\left(1-e^{-s T}\right) V_{R}(s) .
\end{aligned}
$$

Substituting this result into (24), the following formula is obtained:

$$
I_{R}(s)=V_{R}(s) Z_{R}^{-1}(s),
$$

where after neglecting the small parameter $\xi_{n}^{*} / \tau<T / \tau \ll 1$, the inverse arc impedance becomes

$$
\begin{aligned}
Z_{R}^{-1}(s) & =\left(1-e^{-s T}\right) \sum_{n=0}^{\infty} e^{-s T n} \bar{g}\left(T n+\xi_{n}^{*}\right) \\
& \approx G_{0}\left(1-e^{-s T}\right) \sum_{n=0}^{\infty} e^{-s T n}\left(1-e^{-T n / \tau}\right) \\
& =\frac{G_{0}\left(1-e^{-T / \tau}\right)}{e^{s T}-e^{-T / \tau}} .
\end{aligned}
$$

Then, substituting (28) into (22), the electric arc reflection coefficient $\Gamma_{R}$ is obtained in the form

$$
\Gamma_{R}(s)=\frac{e^{s T}-e^{-T / \tau}-G_{0} Z_{0}\left(1-e^{-T / \tau}\right)}{e^{s T}-e^{-T / \tau}+G_{0} Z_{0}\left(1-e^{-T / \tau}\right)} .
$$

Since the fault conductance is small, that is, $G_{0} Z_{0} \ll 1$, then the following asymptotic formula can be obtained by expanding (29) in power series in $G_{0} Z_{0}$ :

$$
\Gamma_{R}(s)=1-\frac{2 G_{0} Z_{0}\left(1-e^{-T / \tau}\right)}{e^{s T}-e^{-T / \tau}} .
$$


This formula should be substituted into (19). The inverse Laplace transform of (19) will yield the solution to the transmission line equations in the time domain. For calculating the inverse Laplace transform with (30), it should be mentioned that the reflection coefficient (30) has an infinite number of poles of order 1:

$$
s=-\tau^{-1}+j n \omega, \quad n \in \mathbf{Z} .
$$

On the other hand, the same function can be expanded in the following analytical series:

$$
\Gamma_{R}(s)=1-2 G_{0} Z_{0}\left(1-e^{-T / \tau}\right) \sum_{n=0}^{\infty} e^{-s(n+1) T} e^{-n T / \tau} .
$$

Either formula (30) or formula (32) can be used in (19).

The electric arc is an essentially nonlinear effect in the sense that the relation between the arc voltage and current is nonlinear. Therefore, the arc impedance should depend explicitly or implicitly on the arc voltage. Moreover, its form can be quite different for varying operational regimes on the transmission line. Indeed, one can observe that the arc impedance (28) and the reflection coefficient (32), respectively, contain the information about the arc voltage through the period of oscillations. On the other hand, though formulae (28), (30), and (32) are inapplicable for the arc voltage of an arbitrary form, they are suitable for an arbitrary periodical voltage, because only the assumption of a general periodic voltage form has been used in the arc impedance deduction.

The correctness of the arc impedance formula (28) can be demonstrated by transforming to the time domain (27) considering a sinusoidal voltage. As a result, after expanding (28) in series, one can obtain, by calculating the respective residues,

$$
\begin{aligned}
i_{R}(t)= & V_{0} G_{0}\left(1-e^{-T / \tau}\right) \sum_{n=0}^{\infty} e^{-T n / \tau} \\
& \times \frac{1}{2 \pi j} \int_{-j \infty+\alpha}^{+j \infty+\alpha} e^{s(t-T(n+1))} \frac{\omega}{\omega^{2}+s^{2}} d s \\
= & V_{0} G_{0}\left(1-e^{-T / \tau}\right) \sin (\omega t) \sum_{n=0}^{\infty} e^{-T n / \tau} \theta(t-T(n+1)) \\
= & V_{0} G_{0}\left(1-e^{-T / \tau}\right) \sin (\omega t) \sum_{n=0}^{[t / T]-1} e^{-T n / \tau} \theta(t-T) \\
= & v_{R}(t) G_{0}\left(1-e^{-[t / T](T / \tau)}\right) \theta(t-T)
\end{aligned}
$$

where $\theta(t-T(n+1))$ is the Heaviside step function and $[t / T]$ is the integer part of $t / T$. Then, the arc conductance becomes

$$
\begin{aligned}
\bar{g}(t) & =G_{0}\left(1-e^{-[t / T](T / \tau)}\right) \theta(t-T) \\
& =G_{0}\left(1-e^{-[t / T](T / \tau)}\right) \theta(t) .
\end{aligned}
$$

This conductance has a ladder form. A small difference between the behavior of the conductance (34) and (9) is originated by the approximation used in the arc impedance deduction (28). To avoid this error in the inverse Laplace transform calculation, a "smoothing operator" $\mathbf{S}$, which transforms a discontinuous function and its derivative into smooth functions, can be introduced. Indeed, if $\phi(t)$ is a smooth function, then, the operator $\mathbf{S}$ can be defined as follows:

$$
\mathbf{S}: \phi^{(k)}\left(\left[\frac{t}{T}\right] \cdot T\right) \longmapsto \phi^{(k)}(t), \quad \text { where } k=0,1 .
$$

Such definition of the smoothing operator, which includes the smoothing of the generalized functions such as the first derivative $\phi^{\prime}([t / T] \cdot T)$, is required in order to reach the commutation between $\mathbf{S}$ and the derivative operator; that is,

$$
\mathbf{S}\left(\frac{d}{d t}\right)=\left(\frac{d}{d t}\right) \mathbf{S}
$$

Applying this operator to the ladder-form conductance (34), we finally obtain the smooth arc conductance (9). Thus, the arc impedance and the arc reflection coefficient take the form

$$
\begin{aligned}
Z_{R}^{-1}(s) & =\mathbf{S} \frac{G_{0}\left(1-e^{-T / \tau}\right)}{e^{s T}-e^{-T / \tau}}, \\
\Gamma_{R}(s) & \approx 1-\mathbf{S} \frac{2 G_{0} Z_{0}\left(1-e^{-T / \tau}\right)}{e^{s T}-e^{-T / \tau}},
\end{aligned}
$$

where the smoothing operator should be applied after the inverse Laplace transform.

\section{Interaction between the HIF Model and the Transmission Line}

5.1. The HIF Model and the Transmission Line. For modeling the interaction between the transmission line and the HIF, we should substitute the reflection coefficient (37) into solution (19) to the transmission line equation, with the respective initial conditions. According to usual assumptions [22], the source impedance in Figure 3 can be taken as $Z_{S}=Z_{0}$ and therefore the generator reflection coefficient is zero; that is, $\Gamma_{S}(s)=0$. Then, (19) is significantly simplified:

$$
\begin{aligned}
V(x, s)= & \frac{1}{2}\left(V_{s}(s)-\frac{Z_{0} i(0,0)}{s}\right)\left(e^{(-s / c) x}+\Gamma_{A}(s) e^{(-s / c)(2 l-x)}\right) \\
& +e^{(-s / c)(l-x)}\left(\frac{1+\Gamma_{R}(s)}{2} \frac{Z_{0} i(l, 0)}{s}\right. \\
& +\frac{1}{2 s c} \int_{0}^{l}\left(e^{(s / c)(l-y)}+\Gamma_{A}(s) e^{(-s / c)(l-y)}\right) \\
& \left.\times\left(v_{t}(y, 0)+s v(y, 0)\right) d y\right) \\
& -\frac{1}{s c} \int_{0}^{x} \sinh \left(\frac{s(x-y)}{c}\right)\left(v_{t}(y, 0)+s v(y, 0)\right) d y .
\end{aligned}
$$


It is assumed that before the HIF has occurred, the line has been energized and operating in normal conditions. Then, the fault occurs at $t=0$. To reduce the effect of the load on the transmission line, the approximation of a semi-infinite line is considered. Thus, the voltage of the form $v(x, 0)=$ $\left(V_{0} / 2\right) \sin (\omega(t-x / v))$ was propagating in the line before $t=0$, and therefore the following initial conditions should be taken in (38):

$$
\begin{gathered}
v(x, 0)=-\frac{V_{0}}{2} \sin \left(\frac{\omega x}{c}\right), \quad i(0,0)=0 \\
v_{t}(x, 0)=\frac{\omega V_{0}}{2} \cos \left(\frac{\omega x}{c}\right), \quad i(l, 0)=-\frac{V_{0}}{2 Z_{0}} \sin \left(\frac{\omega l}{c}\right) .
\end{gathered}
$$

Then, by substituting (37) and (39) into (38) and calculating the inverse Laplace transform, the solution (38) in the time domain takes the form

$$
\begin{aligned}
v(x, t)= & \frac{V_{0}}{2} \sin \left[\omega\left(t-\frac{x}{c}\right)\right] \\
& +\frac{V_{0}}{2}\left\{1-2 G_{0} Z_{0}\left(1-e^{(-1 / \tau)(t-(l-x) / c)}\right)\right\} \\
& \times \sin \left[\omega\left(t-\frac{2 l-x}{c}\right)\right] \theta\left(t-\frac{l-x}{c}\right),
\end{aligned}
$$

where $\theta(t-(l-x) / c)$ is the unit-step function.

5.2. Single-Pulse Propagation. After a time interval greater than the time of arc relaxation $\tau$, the oscillations in the transmission line reach steady state conditions given by the following equation:

$$
\begin{aligned}
v_{s t}(x, t)=\frac{V_{0}}{2}\{ & \sin \left[\omega\left(t-\frac{x}{c}\right)\right] \\
& \left.+\left(1-2 G_{0} Z_{0}\right) \sin \left[\omega\left(t-\frac{x}{c}-\frac{2 l}{c}\right)\right]\right\}
\end{aligned}
$$

which is obtained from (40) by considering $t \rightarrow \infty$. Then, at a given moment $t_{0} \gg \tau$, a single pulse can be applied at the source bus and the signal is reflected back by the HIF and measured at the sending end. By analyzing the time of return and form of the reflected signal, it is possible to know the fault characteristics and the distance at which the fault has occurred.

To analyze the pulse propagation, the solution to (19) can be represented as a superposition of the steady state solution (41) and the propagating pulse:

$$
\begin{aligned}
& v(x, t)=v_{s t}(x, t)+\varphi(x, t), \\
& i(x, t)=i_{s t}(x, t)+\eta(x, t) .
\end{aligned}
$$

Since the steady state (41) is the solution to (19) (and to (38) consequently) with the initial conditions (39), then, by substituting (42) into (38), we can obtain the respective equation for the component $\Phi(x, s)$ :

$$
\begin{aligned}
\Phi(x, s)= & -\frac{1}{2}\left(e^{(-s / c) x}+\Gamma_{R}(s) e^{(-s / c)(2 l-x)}\right) \frac{Z_{0}}{s} \eta\left(0, t_{0}\right) \\
& +e^{(-s / c)(l-x)} \\
& \times\left(\frac{1+\Gamma_{R}(s)}{2} \frac{Z_{0}}{s} \eta\left(l, t_{0}\right)\right. \\
& +\frac{1}{2 s c} \int_{0}^{l}\left(e^{(s / c)(l-y)}+\Gamma_{R}(s) e^{(-s / c)(l-y)}\right) \\
& \quad \frac{1}{s c} \int_{0}^{x} \sin h\left(\frac{s(x-y)}{c}\right) \\
& \times\left(\varphi_{t}\left(y, t_{0}\right)+s \varphi\left(y, t_{0}\right)\right) d y,
\end{aligned}
$$

where $t=t_{0}$ is taken as the initial moment. The initial conditions for the component $\eta(x, t)$ at the ends of the line must be equal to zero. We should note that (38) is linear just formally. In fact, the nonlinearity of (38) consists in the fact that the steady state component and the perturbed part $\varphi(x, t)$ are not independent. The dependence of the perturbed component $\varphi(x, t)$ on the steady state oscillations is expressed in (43) through the reflection coefficient $\Gamma_{R}(s)$.

The initial condition for (43) is given by the Dirac deltafunction [23] with a constant $E$ :

$$
\varphi\left(x, t_{0}\right)=E \delta(x) \text {. }
$$

Since the transmission line model under consideration is lossless, then the pulse propagates to the point of fault as a simple travelling wave:

$$
\begin{aligned}
& \varphi(x, t)=E \delta\left(x-c\left(t-t_{0}\right)\right), \\
& \varphi_{t}(x, t)=-c E \delta^{\prime}\left(x-c\left(t-t_{0}\right)\right) .
\end{aligned}
$$

Then, the second initial condition for (43) is

$$
\varphi_{t}\left(x, t_{0}\right)=-c E \delta^{\prime}(x) .
$$

Substituting the initial conditions (44) and (46) into (43), the following expression is obtained:

$$
\Phi(x, s)=\frac{E}{c}\left(e^{-s x / c}+\Gamma_{R}(s) e^{(-2 s l / c)+(s x / c)}\right) .
$$

By applying the inverse Laplace transform to (47) with the reflection coefficient (37) expanded in series (32), it can be found in the time domain:

$$
\begin{aligned}
\varphi(x, t)= & E\left\{\delta\left(x-c\left(t-t_{0}\right)\right)+\delta\left(2 l-x-c\left(t-t_{0}\right)\right)\right\} \\
& -\frac{2 E G_{0} Z_{0}}{c}\left(1-e^{-T / \tau}\right) \mathbf{S} \\
& \times \sum_{n=0}^{\infty} e^{-T n / \tau} \delta\left(t-t_{0}+\frac{x}{c}-\frac{2 l}{c}-T(n+1)\right) .
\end{aligned}
$$


Let us denote

$$
t-t_{0}+\frac{x}{c}-\frac{2 l}{c} \equiv q .
$$

Then, applying the commutation relation (36) one can obtain, similarly to (34),

$$
\begin{aligned}
\mathbf{S} & \sum_{n=0}^{\infty} e^{-T n / \tau} \delta(q-T(n+1)) \\
& =\mathbf{S} \frac{d}{d q} \sum_{n=0}^{\infty} e^{-T n / \tau} \theta(q-T(n+1)) \\
& =\mathbf{S} \frac{d}{d q} \sum_{n=0}^{[q / T]-1} e^{-T n / \tau} \theta(q-T) \\
& =\mathbf{S} \frac{d}{d q}\left(\frac{1-e^{-[q / T] T / \tau}}{1-e^{-T / \tau}} \theta(q-T)\right) \\
& =\frac{1}{1-e^{-T / \tau}} \frac{d}{d q} \mathbf{S}\left(1-e^{-[q / T] T / \tau}\right) \theta(q-T) \\
& =\frac{1}{\tau} \frac{e^{-q / \tau}}{1-e^{-T / \tau}} \theta(q) .
\end{aligned}
$$

By substituting this result into (48), the single-pulse propagation in the time domain is finally obtained:

$$
\begin{aligned}
\varphi(x, t)= & E\left\{\delta\left(x-c\left(t-t_{0}\right)\right)+\delta\left(2 l-x-c\left(t-t_{0}\right)\right)\right\} \\
& -\frac{2 E G_{0} Z_{0}}{c \tau} \exp \left\{-\frac{1}{\tau}\left(t-t_{0}+\frac{x}{c}-\frac{2 l}{c}\right)\right\} \\
& \times \theta\left(t-t_{0}+\frac{x}{c}-\frac{2 l}{c}\right) .
\end{aligned}
$$

From (51) it follows that the returned signal is a pulse decreasing exponentially, which can provide information about the HIF.

\section{Computer Simulations}

In this section, several computer simulations and measured results are presented. The aim of the first and second cases of study is to validate the HIF model developed in order to analyze the interaction between the arc conductance and the transmission line at power frequency. The objective of the third case of study is to analyze the model performance at higher frequencies, during a steep fronted pulse applied at the substation bus.

6.1. Case 1. In this first case of study, a comparison between the arc conductance measured during a HIF and the arc conductance calculated with the developed model is presented. The respective constants involved in (9) were calculated from field measurements in Section 2.1, and they are $G_{0}=$ $0.000932 \mho$ and $\tau=0.0298 \mathrm{~s}$. Figure 4 shows the experimental arc conductance obtained from field measurements and the arc conductance calculated by using (9).

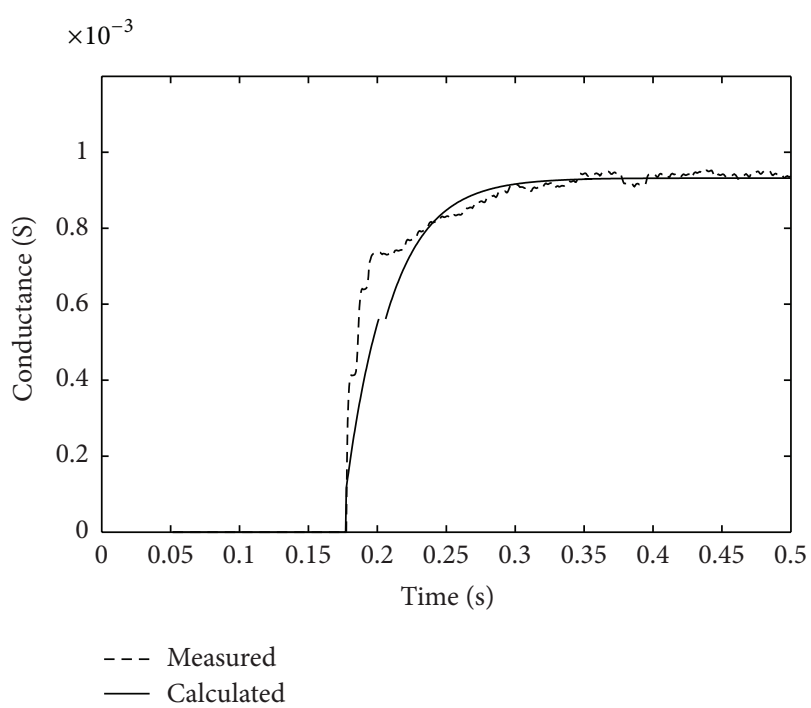

FIGURE 4: Measured and calculated conductance during a HIF.

A close comparison between measured and calculated results for the arc conductance, a key parameter for modeling HIFs, shows a good agreement in magnitude and general trend of waveforms. The error was calculated by using the following equation:

$$
\text { error }=\frac{\sqrt{\sum_{k=1}^{n}\left|g_{k}-g_{k}^{M}\right|^{2}}}{\sqrt{\sum_{k=1}^{n}\left|g_{k}\right|^{2}}} \text {, }
$$

where $g_{k}$ is the calculated conductance and $g_{k}^{M}$ is the measured conductance. In general, the error in magnitude is smaller than $5 \%$. These results show that the assumptions made for the development of the HIF are correct and that the model is capable of calculating the arc conductance during a HIF.

6.2. Case 2. Once the arc conductance has been calculated, in this second case of study, a comparison between voltages and currents measured and calculated using the HIF model is carried out.

The HIF was applied at the end of a $0.5 \mathrm{~km}$ unloaded distribution feeder. The substation bus voltage is $V_{0}$ at point $x=0$. The characteristic impedance of the line is taken as $Z_{0}=Z_{S}$. The respective parameters involved in the solution of (40) are given in Table 1 . Figure 5 shows the measured and calculated voltages at the substation bus by using (40).

In Figure 5 the HIF fault is applied at $t=0.177 \mathrm{~s}$, and there are no significant changes on voltages magnitude or distortion; they are practically superimposed. In brief, there exists good agreement between measured and calculated voltages. This voltage behavior is explained by the fact that voltage at the substation bus is mainly dependent on the source power, not from the HIF located at $0.5 \mathrm{~km}$.

Figure 6 shows the current wave measured and calculated at $x=0$, with magnitudes of about $8 \mathrm{~A}$ rms. The measured current wave has some harmonic distortion due to the 
TABLE 1: Parameters for the first and second case of study.

\begin{tabular}{lcc}
\hline Parameter & Value & \\
\hline$G_{0}$ & 0.000932 & $\mho$ \\
$\tau$ & 0.0298 & $\mathrm{~S}$ \\
$V_{0}$ & 11267 & $\mathrm{~V}$ \\
$c$ & 299792 & $\mathrm{~km} / \mathrm{s}$ \\
$l$ & 0.5 & $\mathrm{~km}$ \\
$\omega$ & 377 & $\mathrm{rad} / \mathrm{s}$ \\
\hline
\end{tabular}

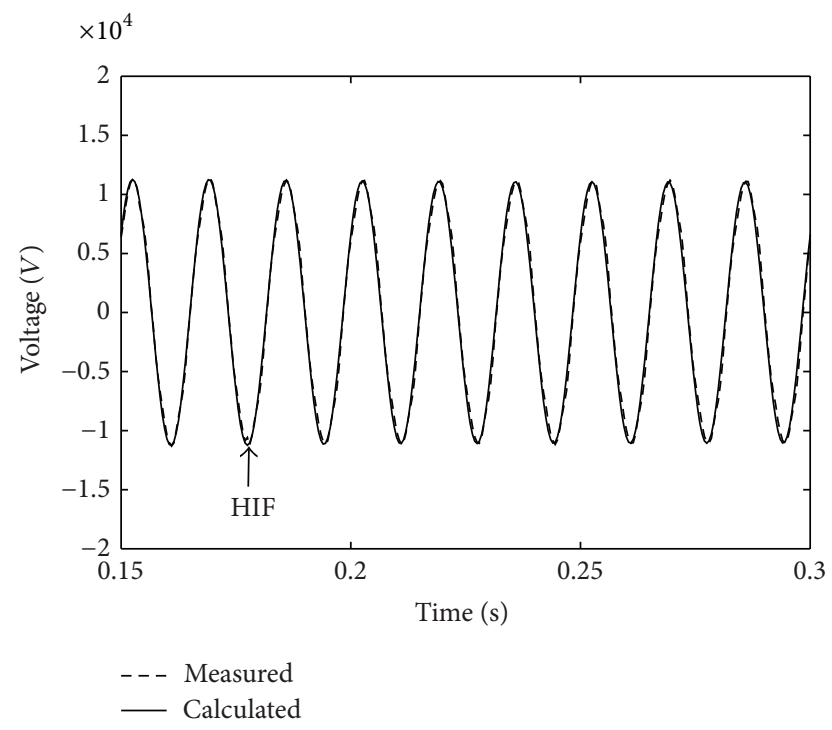

FIGURE 5: Measured and calculated voltage during a HIF.

nonlinear behavior of the arc conductance. No harmonic distortion exists in the current waveform calculated because of the assumptions made for developing the HIF model, mainly the use of average values.

Observe in Figures 4 and 6 that after about $3.3 \tau$ the arc conductance and the current wave measured and calculated reach their steady state. This behavior is due to the initial arc conductance, which is highly nonlinear and the current measured at the substation bus is dependent on this arc conductance since the line is unloaded. Then, after some time has elapsed, the electric arc associated with the HIF is stabilized and the arc conductance reaches its steady state value. Taking into account the HIF model assumptions, it is possible to establish that there exists good agreement between the general trends of the measured and calculated results for current waveforms.

Cases 1 and 2 demonstrate that the proposed model is capable of analyzing the dynamic interaction between the distribution line and the arc conductance associated with HIFs at power frequency.

6.3. Case 3. In order to analyze the interaction between the arc conductance and the HIF at higher frequencies, a steep

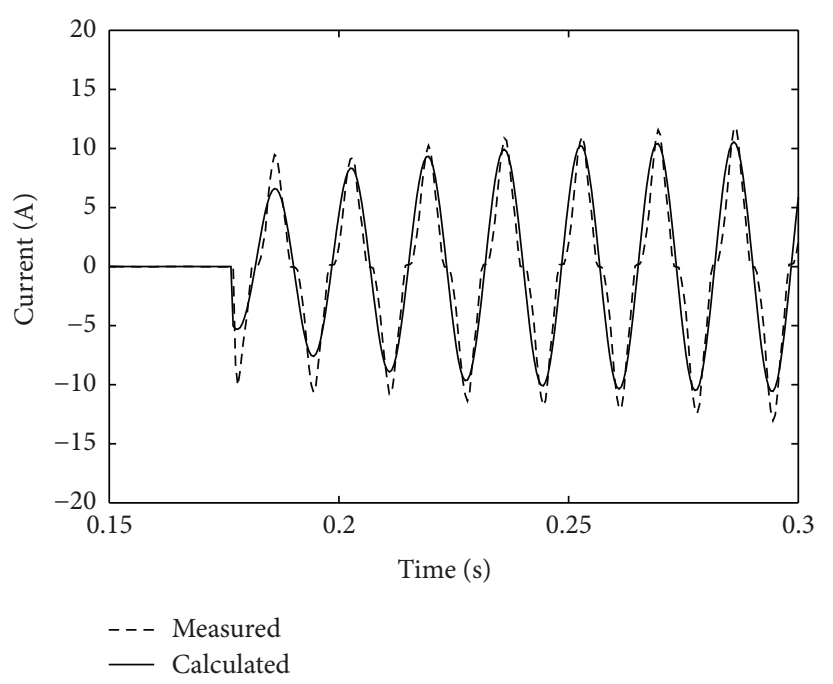

FIgURE 6: Measured and calculated current during a HIF.

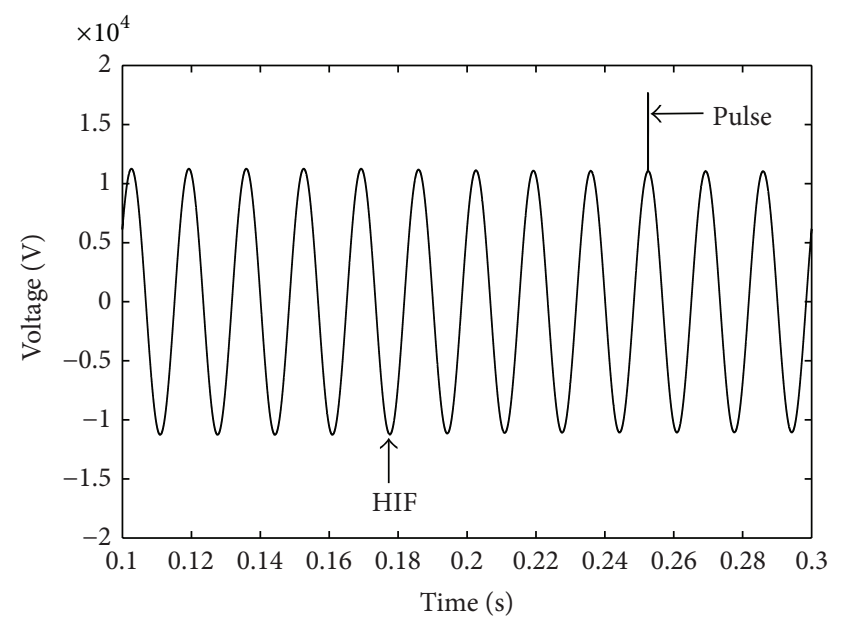

FIGURE 7: Forward and reflected voltage pulses from the HIF.

fronted pulse is applied during a HIF. The pulse signal $E \delta(x-$ $\left.c\left(t-t_{0}\right)\right)$ was modelled by a Gaussian function of the type:

$$
\frac{E}{\sqrt{2 \pi} \sigma_{x}} \exp \left(-\frac{\left(x-v\left(t-t_{0}\right)\right)^{2}}{2 \sigma_{x}^{2}}\right)
$$

where the pulse constant $E$ and the signal width $\sigma_{x}$ were taken as $E=50 \mathrm{kV} \cdot \mathrm{km}$ and $\sigma_{x}=0.1 \mathrm{~km}$. The sequence of events is as follows: first, a HIF occurs at $t=0.177 \mathrm{~s}$. in a $0.5 \mathrm{~km}$ distribution feeder and then at $t=0.252 \mathrm{~s}$. a single pulse is applied. Figure 7 shows the forward and reflected pulse from the HIF superimposed over the steady state voltage (41).

In Figure 8, the forward and reflected pulses have been separated from the steady state voltage. Some additional information can be extracted from Figure 8. For example, let us denote $\Delta t$ by the time interval between the two pulses. 


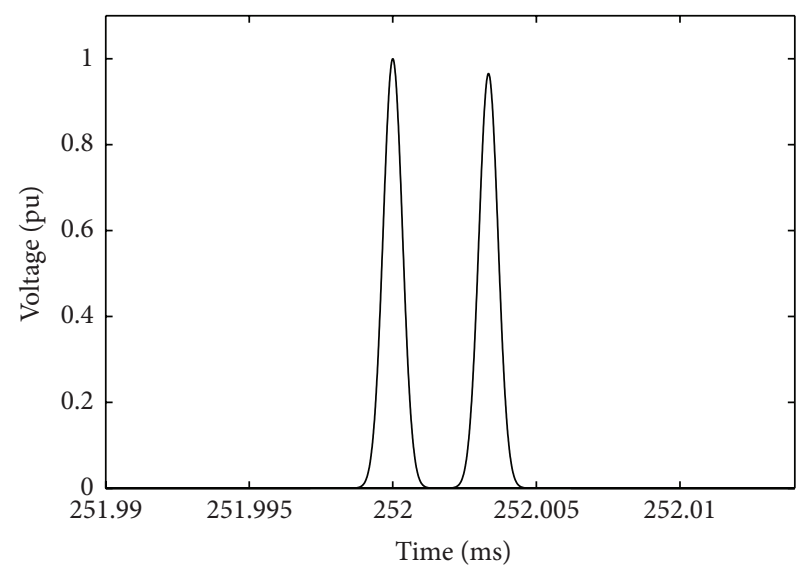

Figure 8: Forward and reflected pulse voltage due to HIF.

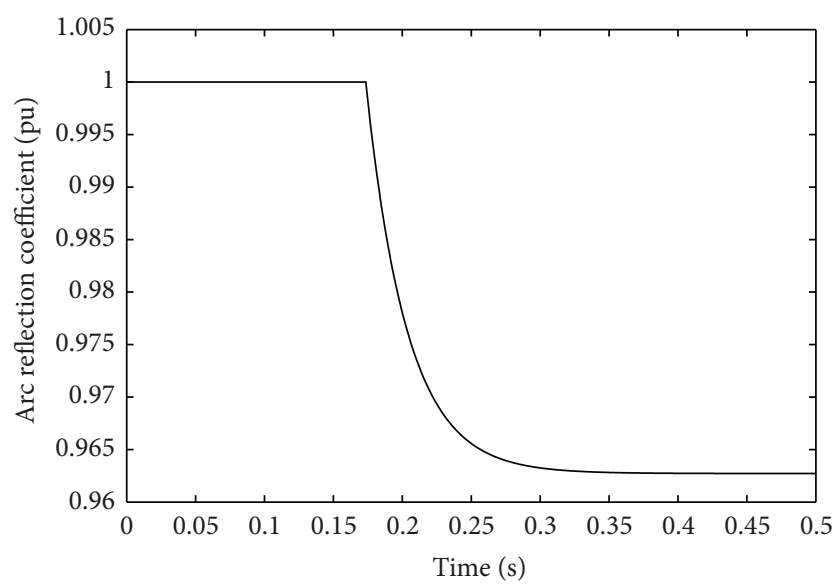

FIGURE 9: Calculated time dependent reflection coefficient.

Then, the distance to the HIF can be calculated according to the formula

$$
l=\frac{c \Delta t}{2}
$$

where $c$ is the wave velocity in the transmission line.

Observe that the reflected pulse is smaller than the applied one. This decrease in pulse magnitude is due to the fact that the reflection coefficient is less than one and part of the pulse energy is dissipated through the electric arc. Figure 9 shows the voltage reflection coefficient calculated in the time domain using (22). As expected, this coefficient is dependent on the arc conductance, which is varying with time after fault insertion. Before fault inception the reflection coefficient is equal to one because the line is in open circuit conditions. After fault inception the magnitude for the reflected pulse from the HIF is $0.96 \mathrm{pu}$, for an applied pulse at $t=0.252 \mathrm{~s}$ and magnitude equals $1 \mathrm{pu}$.

The results obtained in the three cases of study are in full agreement with transmission line theory but include a HIF at the end of the distribution feeder, which is represented by a time varying conductance due to the associated electric arc.
In brief, the three cases of study describe the interaction between the transmission line and the electric arc associated with HIFs at power and higher frequencies with an acceptable accuracy.

\section{Conclusions}

It is well known that HIF depends on the type of ground surface, moisture, conductor type, environmental conditions, and voltage level. Many HIFs have similar characteristics that can be represented considering variations on the arc parameters like conductance and time constant.

In this work, an analytical model for analyzing the interaction between HIFs and a transmission line has been developed. The analysis of this interaction involves the joint solution of a partial differential equation and a linear differential equation. The solution of this system is complex, but the mathematical methods used in this paper lead to an elegant solution to the problem with no significant loss of accuracy. The solution is based on considering that for periodic voltages with a period smaller than the arc time constant, the electric arc can be represented as load impedance. Several expressions that relate the relevant arc and transmission line parameters were obtained, leading to recursive equations that calculate current and voltage along the line, as well as the dynamic of the electric arc associated with the HIF.

Three cases of study were presented. The aim of the first two cases is to validate the model. In this sense, current and voltages measured during a HIF were compared with the calculated results provided by the analytical model. The comparison was extended to the arc conductance during a HIF. The comparison shows good agreement between measured and calculated results, which demonstrate that the proposed model is capable of analyzing the interaction between the transmission line and the electric arc associated with the HIF.

In the third case of study a voltage pulse was applied to the distribution feeder during a HIF. The obtained results are similar to those predicted by transmission line theory. There exists a forward pulse along the transmission line which is reflected back by the HIF. The reflected pulse is smaller than the applied pulse because of the reflection coefficient between the transmission line and the electric arc conductance. This reflection coefficient changes dynamically because the arc conductance changes with time. Also, considering the arrival times for the forward and reflected pulse, the distance to the fault can be calculated. Of course, this is a simplified case, where the author's interest is to develop a model for analyzing the interaction between the electric arc and the transmission line and not to develop a technique for HIF localization.

The authors consider that an analytical model for analyzing the interaction between the electric arc associated with HIF and a transmission line has been presented. The model has been validated and the computer results show good agreement with measured results. Also, a general description of the basic foundation of this complex interaction has been presented which enhances the understanding of the HIF 
phenomenon. Future works should apply the model in more complex scenarios for HIF localization purposes.

\section{Conflict of Interests}

The authors declare that there is no conflict of interests regarding the publication of this paper.

\section{References}

[1] J. Tengdin, R. Westfall, K. Stephan et al., "High impedance fault detection technology," IEEE Power System Relaying Committee Working Group D15 Report, 1996, http:/grouper.ieee .org/groups/td/dist/documents/highz.pdf.

[2] C.-L. Huang, H.-Y. Chu, and M.-T. Chen, "Algorithm comparison for high impedance fault detection based on staged fault test," IEEE Transactions on Power Delivery, vol. 3, no. 4, pp. 14271435, 1988.

[3] A. E. Emanuel, D. Cyganski, S. Shiller, J. A. Orr, and E. M. Gulachenski, "High impedance fault arcing on sandy soil in $15 \mathrm{kV}$ distribution feeders: contributions to the evaluation of the low frequency spectrum," IEEE Transactions on Power Delivery, vol. 5, no. 2, pp. 676-686, 1990.

[4] A. A. Girgis, W. Chang, and E. B. Makram, "Analysis of highimpedance fault generated signals using a Kalman filtering approach," IEEE Transactions on Power Delivery, vol. 5, no. 4, pp. 1714-1724, 1990.

[5] S. Ebron, D. L. Lubkeman, and M. White, "Neural network approach to the detection of incipient faults on power distribution feeders," IEEE Transactions on Power Delivery, vol. 5, no. 2, pp. 905-914, 1990.

[6] J. Stoupis, M. Maharsi, R. Nuqui, S. A. Kunsman, and R. Das, "Ground alert: reliable detection of high-impedance faults caused by downed conductors," ABB Review, no. 1, pp. 28-32, 2004.

[7] M. Adamiak, C. Wester, M. Thakur et al., "High impedance fault detection on distribution feeders," Protection and Control Journal GE, pp. 25-31, 2006.

[8] D. Hou, "Detection of high-impedance faults in power distribution systems," in Proceedings of the 33rd Annual Western Protective Relay Conference, Spokane, Wash, USA, 2006.

[9] V. Torres, J. L. Guardado, H. F. Ruiz, and S. Maximov, "Modeling and detection of high impedance faults," International Journal of Electric Power and Energy Systems, vol. 61, pp. 163-172, 2014.

[10] C. H. Kim, H. Kim, Y. H. Ko, S. H. Byun, R. K. Aggarwal, and A. T. Johns, "A novel fault-detection technique of high-impedance arcing faults in transmission lines using the wavelet transform," IEEE Transactions on Power Delivery, vol. 17, no. 4, pp. 921-929, 2002.

[11] V. Torres and H. F. Ruiz, "High impedance fault detection in distribution system using wavelet transform," in Proceedings of the 8th IEEE International Conference on Electrical Engineering Computing Science and Automatic Control (CCE), pp. 4-11, Merida City, Mexico, September 2011.

[12] V. Torres and H. F. Ruiz, "High impedance fault detection using DWT," in Proceedings of the IEEE Electronics, Robotic and Automotive Mechanics Conference (CERMA '11), Cuernavaca Morelos, Mexico, November 2011.

[13] N. Faridnia, H. Samet, and B. D. Dezfuli, "A new approach to high impedance fault detection based on correlation functions," in Artificial Intelligence Applications and Innovations, vol. 381, pp. 453-462, Springer, 2012.

[14] M. B. Djurić, Z. M. Radojević, and V. V. Terzija, "Numerical algorithm for arcing faults detection and fault distance calculation on overhead lines," Electric Machines and Power Systems, vol. 25, no. 9, pp. 939-953, 1997.

[15] M. Chen, J. Zhai, Z. Lang et al., "High impedance fault location in transmission line using nonlinear frequency analysis," in Life System Modeling and Intelligent Computing, vol. 6328, pp. 104111, Springer, 2010.

[16] V. Terzija, N. I. Elkalashy, G. Preston, V. Stanojevič, and G. Štrbac, "Detection of arcing faults: modelling, simulation, testing and algorithms aspects," in Proceeding of the IEEE Lausanne Power Tech Conference, pp. 1147-1152, Lausanne, Switzerland, July 2007.

[17] A. M. Cassie, "Theorie nouvelle des arcs de rupture et de la rigidité des circuits," Cigre Report, vol. 102, pp. 588-608, 1939.

[18] O. Mayr, "Beiträge zur theorie des statischen und des dynamischen lichtbogens," Archiv für Elektrotechnik, vol. 37, no. 12, pp. 588-608, 1943.

[19] S. Maximov, V. Venegas, J. L. Guardado, E. Melgoza, and D. Torres, "Asymptotic methods for calculating electric arc model parameters," Electrical Engineering, vol. 94, no. 2, pp. 89-96, 2012.

[20] J. L. Guardado, S. G. Maximov, E. Melgoza, J. L. Naredo, and P. Moreno, "An improved arc model before current zero based on the combined Mayr and Cassie arc models," IEEE Transactions on Power Delivery, vol. 20, no. 1, pp. 138-142, 2005.

[21] M. Kizilcay and T. Pniok, "Digital simulation of fault arcs in power systems," Europe Transaction on Electrical Power System, vol. 4, no. 3, pp. 55-59, 1991.

[22] J. D. Glover and M. Sarma, "Power system analysis and design," in Transmission Lines Transient Operation, pp. 629-632, PWSKent Publishing, 1989.

[23] V. S. Vladimirov, Equations of Mathematical Physics, Mir Publishers, Moscow, Russia, 1984. 


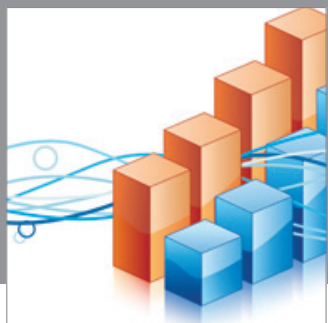

Advances in

Operations Research

mansans

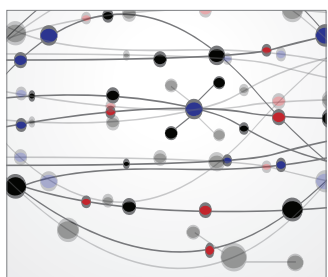

The Scientific World Journal
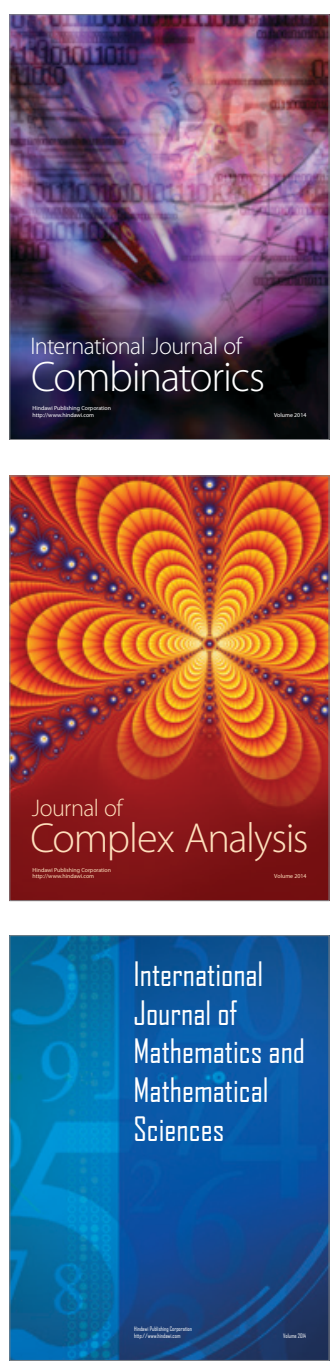
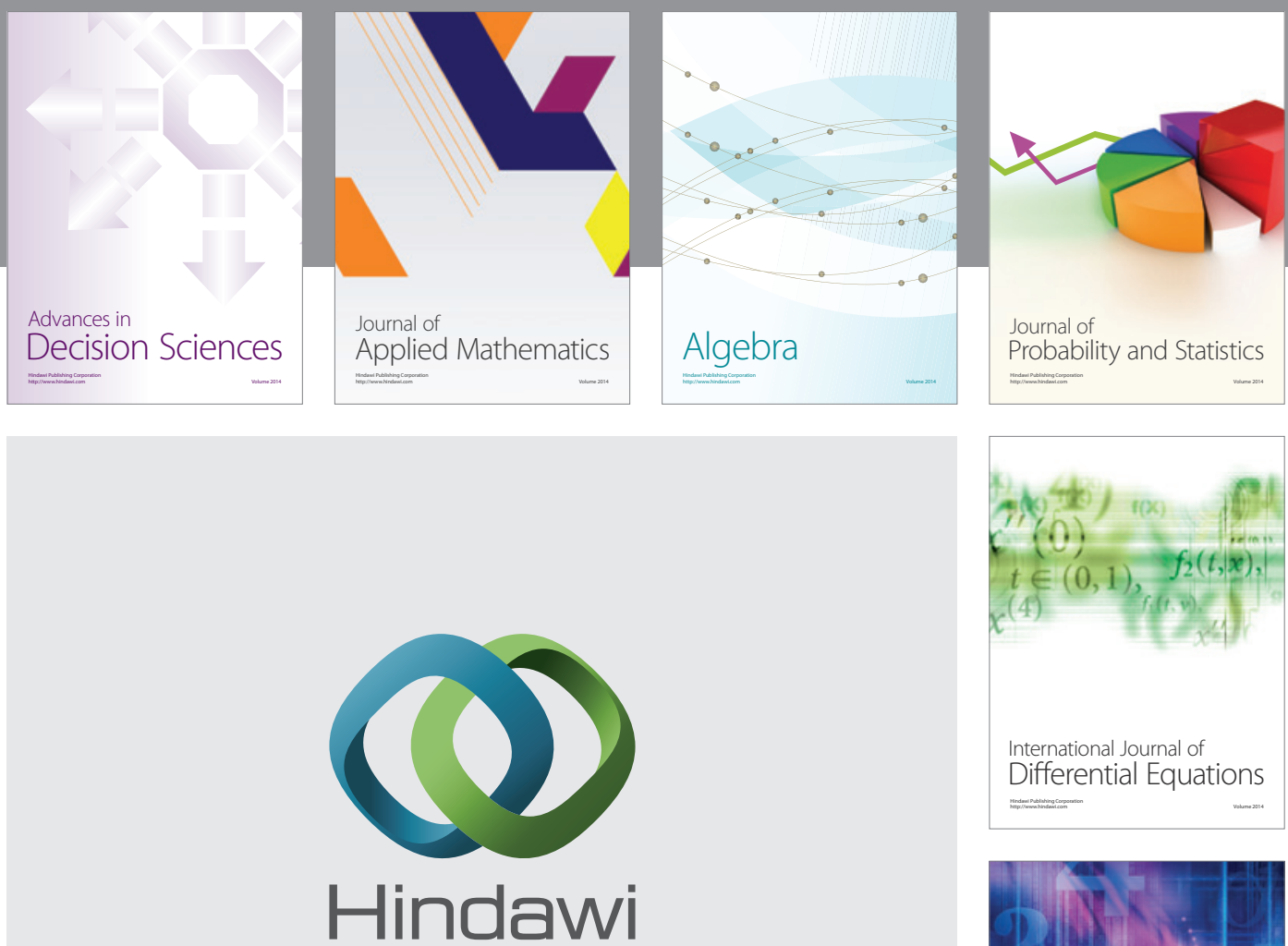

Submit your manuscripts at http://www.hindawi.com
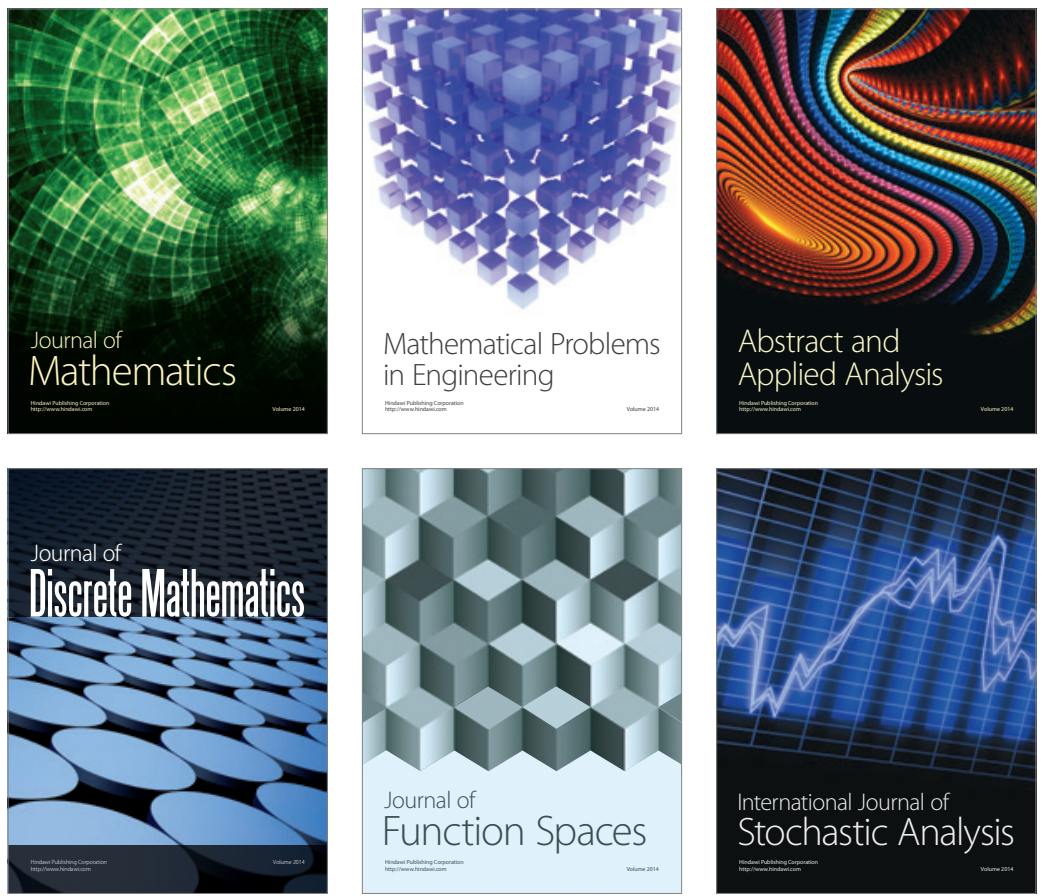

Journal of

Function Spaces

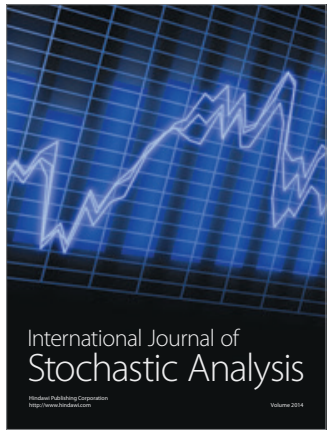

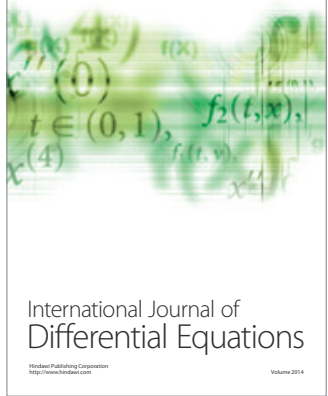
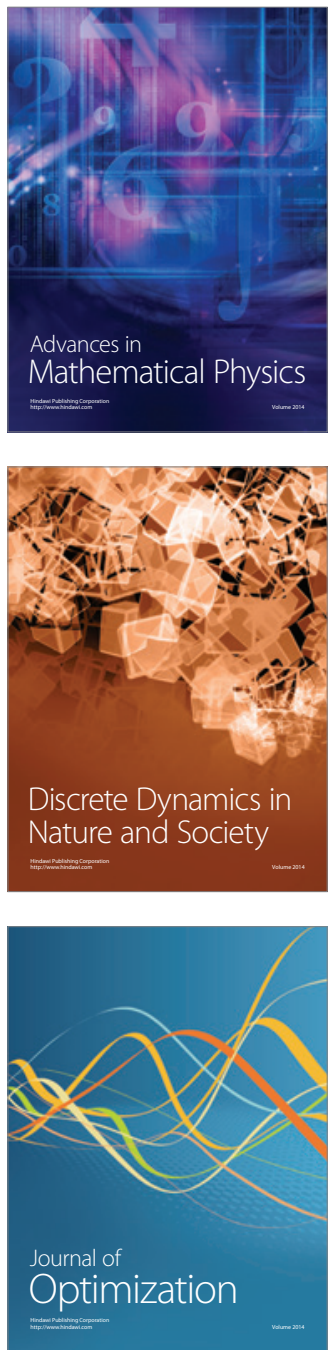